\title{
Entire Fredholm determinants for Evaluation of Semi-classical and Thermodynamical Spectra
}

\author{
Predrag Cvitanović and Gábor Vattayt \\ Niels Bohr Institute, Blegdamsvej 17, DK-2100 Copenhagen Ø, Denmark
}

(November 10, 2018)

\begin{abstract}
Proofs that Fredholm determinants of transfer operators for hyperbolic flows are entire can be extended to a large new class of multiplicative evolution operators. We construct such operators both for the Gutzwiller semi-classical quantum mechanics and for classical thermodynamic formalism, and introduce a new functional determinant which is expected to be entire for Axiom A flows, and whose zeros coincide with the zeros of the Gutzwiller-Voros zeta function.
\end{abstract}

It has been established recently [1],2,3] that the Gutzwiller-Voros zeta function [4 derived from the Gutzwiller semi-classical trace formula [5] is not an entire function. In this letter we construct a new classical evolution operator (6) whose Fredholm determinant (10) is entire for Axiom A flows, and whose spectrum contains the semi-classical Gutzwiller spectrum. Many physically realistic chaotic scattering systems [6, 7] are of Axiom A type, and for them the new determinant has much better convergence properties than the Gutzwiller-Voros and Ruelle type zeta functions utilized previously [0], 8 .

The main idea, extending the dynamical system to the tangent space of the flow, is suggested by one of the standard numerical methods for evaluation of Lyapunov exponents [9]: start at $x_{0}$ with an initial vector $\xi(0)$, and let the flow transport it to $\xi(t)$ along the trajectory $x(t)=f^{t}\left(x_{0}\right)$. The growth rate of this vector is multiplicative along the trajectory

$$
\frac{\left|\xi\left(t+t^{\prime}\right)\right|}{|\xi(0)|}=\frac{\left|\xi\left(t+t^{\prime}\right)\right|}{|\xi(t)|} \times \frac{|\xi(t)|}{|\xi(0)|},
$$

and can be represented by the trajectory of a "unit" vector $\mathbf{u}(t)$ multiplied by the factor $|\xi(t)| /|\xi(0)|$. For asymptotic times and for almost every initial $\left(x_{0}, \xi(0)\right)$, this factor converges to the leading eigenvalue of the linearized stability matrix for the flow.

We implement this multiplicative evaluation of stability eigenvalues by adjoining 10 the $d$-dimensional transverse tangent space $\xi \in T U_{x}, \xi(x) \cdot \mathbf{v}(x)=0$, to the $(d+1)$-dimensional dynamical evolution space $x \in U \subset$ $\mathbf{R}^{d+1}$. The dynamics in the $(x, \xi) \in U \times T U_{x}$ space is governed by the system of equations of variations [1]:

$$
\dot{x}=\mathbf{v}(x), \quad \dot{\xi}=\mathbf{D} \mathbf{v}(x) \xi .
$$

Here $\mathbf{D} \mathbf{v}(x)$ is the transverse derivative matrix of the flow. We write the solution as

$$
x(t)=f^{t}\left(x_{0}\right), \quad \xi(t)=\mathbf{J}^{t}\left(x_{0}\right) \cdot \xi_{0},
$$

with the tangent space vector $\xi$ transported by the transverse stability matrix $\mathbf{J}^{t}\left(x_{0}\right)=\partial x(t) / \partial x_{0}$. In order to determine the length of the vector $\xi$ we introduce a signed norm, a differentiable scalar function $g(\xi)$ with the property $g(\Lambda \xi)=\Lambda g(\xi)$ for any number $\Lambda$. While in general such norm is a space dependent function $g(\xi, x)$, we shall assume here for reasons of notational simplicity that $g$ is a function of $\xi$ only. An example is the function

$$
g\left(\begin{array}{c}
\xi_{1} \\
\xi_{2} \\
\cdots \\
\xi_{d}
\end{array}\right)=\xi_{d}
$$

Any vector $\xi \in T U_{x}$ can now be represented by the product $\xi=\Lambda \mathbf{u}$, where $\mathbf{u}$ is a "unit" vector in the sense that its signed norm is $g(\mathbf{u})=1$, and the factor

$$
\Lambda^{t}\left(x_{0}, \mathbf{u}_{0}\right)=g(\xi(t))=g\left(\mathbf{J}^{t}\left(x_{0}\right) \cdot \mathbf{u}_{0}\right)
$$

is the multiplicative "stretching" factor introduced in (1)

$$
\Lambda^{t^{\prime}+t}\left(x_{0}, \mathbf{u}_{0}\right)=\Lambda^{t^{\prime}}(x(t), \mathbf{u}(t)) \Lambda^{t}\left(x_{0}, \mathbf{u}_{0}\right)
$$

The $\mathbf{u}$ evolution constrained to $E T_{g, x}$, the space of unit tangent vectors transverse to the flow $\mathbf{v}$, is given by rescaling of (2):

$$
\mathbf{u}^{\prime}=R^{t}(x, \mathbf{u})=\frac{1}{\Lambda^{t}(x, \mathbf{u})} \mathbf{J}^{t}(x) \cdot \mathbf{u} .
$$

Eqs. (2), (4) and (5) enable us to define a multiplicative evolution operator on the extended space $U \times E T_{g, x}$

$$
\mathcal{L}^{t}\left(\mathbf{u}^{\prime}, x^{\prime} ; \mathbf{u}, x\right)=e^{h(x)} \delta\left(x^{\prime}-f^{t}(x)\right) \frac{\delta\left(\mathbf{u}^{\prime}-R^{t}(x, \mathbf{u})\right)}{\left|\Lambda^{t}(x, \mathbf{u})\right|^{\beta-1}}
$$

where $h$ is a function additive along the trajectory, and $\beta$ is a number. This should be contrasted to "thermodynamic" 12,13,14 operators of form 


$$
\mathcal{L}^{t}\left(x^{\prime}, x\right)=e^{h(x)} \delta\left(x^{\prime}-f^{t}(x)\right) \frac{1}{\left|\Lambda^{t}(x)\right|^{\beta-1}},
$$

with $\Lambda^{t}(x)$ an eigenvalue of $\mathbf{J}^{t}(x)$. Such operators are not multiplicative in two or more transverse dimensions, for the simple reason that the eigenvalues of successive stability matrices are in general not multiplicative

$$
\Lambda_{a b} \neq \Lambda_{a} \Lambda_{b}
$$

Here $\mathbf{J}_{a b}=\mathbf{J}_{a} \mathbf{J}_{b}$ is the stability matrix of the trajectory consisting of consecutive segments $a$ and $b, \mathbf{J}_{a}$ and $\mathbf{J}_{b}$ are the stability matrices for these segments separately, and $\Lambda$ 's are their eigenvalues. In particular, this lack of multiplicative property for $\Lambda$ 's had until now frustrated attempts [15] to construct evolution operators whose spectrum contains the semi-classical Gutzwiller spectrum.

In order to derive the trace formula for the operator (6) we need to evaluate $\operatorname{Tr} \mathcal{L}^{t}=\int d x d \mathbf{u} \mathcal{L}^{t}(\mathbf{u}, x ; \mathbf{u}, x)$. The $\int d x$ integral yields [16] a weighted sum over primitive periodic orbits $p$ and their repetitions $r$ :

$$
\begin{aligned}
\operatorname{Tr} \mathcal{L}^{t} & =\sum_{p} T_{p} \sum_{r=1}^{\infty} \frac{e^{r h_{p}} \delta\left(t-r T_{p}\right)}{\left|\operatorname{det}\left(1-\mathbf{J}_{p}^{r}\right)\right|} \Delta_{p, r}, \\
\Delta_{p, r} & =\int_{g} d \mathbf{u} \frac{\delta\left(\mathbf{u}-R^{T_{p} r}\left(x_{p}, \mathbf{u}\right)\right)}{\left|\Lambda^{T_{p} r}\left(x_{p}, \mathbf{u}\right)\right|^{\beta-1}},
\end{aligned}
$$

where $\mathbf{J}_{p}$ is the prime cycle $p$ transverse stability matrix. As we shall see below, $\Delta_{p, r}$ is intrinsic to cycle $p$, and independent of any particular cycle point $x_{p}$.

We note next that if the trajectory $f^{t}(x)$ is periodic with period $T$, the tangent space contains $d$ periodic solutions

$$
\mathbf{e}_{i}(x(T+t))=\mathbf{e}_{i}(x(t)), \quad i=1, \ldots, d,
$$

corresponding to the $d$ unit eigenvectors $\left\{\mathbf{e}_{1}, \mathbf{e}_{2}, \cdots, \mathbf{e}_{d}\right\}$ of the transverse stability matrix, with "stretching" factors (14) given by its eigenvalues

$$
\mathbf{J}_{p}(x) \cdot \mathbf{e}_{i}(x)=\Lambda_{p, i} \mathbf{e}_{i}(x), \quad i=1, \ldots, d .
$$

The $\int d \mathbf{u}$ integral in (7) picks up contributions from these periodic solutions. In order to compute the stability of the $i$-th eigendirection solution, it is convenient to expand the variation around the eigenvector $\mathbf{e}_{i}$ in the stability matrix eigenbasis $\delta \mathbf{u}=\sum \delta u_{\ell} \mathbf{e}_{\ell}$. The variation of the map (5) at a complete period $t=T$ is then given by

$$
\begin{aligned}
\delta R^{T}\left(\mathbf{e}_{i}\right) & =\frac{\mathbf{J} \cdot \delta \mathbf{u}}{g\left(\mathbf{J} \cdot \mathbf{e}_{i}\right)}-\frac{\mathbf{J} \cdot \mathbf{e}_{i}}{g\left(\mathbf{J} \cdot \mathbf{e}_{i}\right)^{2}}\left(\frac{\partial g\left(\mathbf{e}_{i}\right)}{\partial \mathbf{u}} \cdot \mathbf{J} \cdot \delta \mathbf{u}\right) \\
& =\sum_{k \neq i} \frac{\Lambda_{p, k}}{\Lambda_{p, i}}\left(\mathbf{e}_{k}-\mathbf{e}_{i} \frac{\partial g\left(\mathbf{e}_{i}\right)}{\partial u_{k}}\right) \delta u_{k} .
\end{aligned}
$$

The $\delta u_{i}$ component does not contribute to this sum since $g\left(\mathbf{e}_{i}+d u_{i} \mathbf{e}_{i}\right)=1+d u_{i}$ implies $\partial g\left(\mathbf{e}_{i}\right) / \partial u_{i}=1$. Indeed, infinitesimal variations $\delta \mathbf{u}$ must satisfy

$$
g(\mathbf{u}+\delta \mathbf{u})=g(\mathbf{u})=1 \quad \Longrightarrow \quad \sum_{\ell=1}^{d} \delta u_{\ell} \frac{\partial g(\mathbf{u})}{\partial u_{\ell}}=0,
$$

so the allowed variations are of form

$$
\delta \mathbf{u}=\sum_{k \neq i}\left(\mathbf{e}_{k}-\mathbf{e}_{i} \frac{\partial g\left(\mathbf{e}_{i}\right)}{\partial u_{k}}\right) c_{k}, \quad\left|c_{k}\right| \ll 1,
$$

and in the neighborhood of the $\mathbf{e}_{i}$ eigenvector the $\int \mathbf{u}$ integral can be expressed as

$$
\int_{g} d \mathbf{u}=\int \prod_{k \neq i} d c_{k}
$$

Inserting these variations into the $\int d \mathbf{u}$ integral we obtain

$$
\begin{gathered}
\int_{g} d \mathbf{u} \delta\left(\mathbf{e}_{i}+\delta \mathbf{u}-R^{T}\left(\mathbf{e}_{i}\right)-\delta R^{T}\left(\mathbf{e}_{i}\right)+\ldots\right) \\
=\int \prod_{k \neq i} d c_{k} \delta\left(\left(1-\Lambda_{k} / \Lambda_{i}\right) c_{k}+\ldots\right) \\
=\prod_{k \neq i} \frac{1}{\left|1-\Lambda_{k} / \Lambda_{i}\right|},
\end{gathered}
$$

and the $\int d \mathbf{u}$ trace $(7)$ becomes

$$
\Delta_{p, r}=\sum_{i=1}^{d} \frac{1}{\left|\Lambda_{p, i}^{r}\right|^{\beta-1}} \prod_{k \neq i} \frac{1}{\left|1-\Lambda_{p, k}^{r} / \Lambda_{p, i}^{r}\right|} .
$$

The corresponding Fredholm determinant is obtained by observing 16] that the Laplace transform of the trace

$$
\operatorname{Tr} \mathcal{L}(s)=\int_{0_{+}}^{\infty} d t e^{s t} \operatorname{Tr} \mathcal{L}(t)
$$

is a logarithmic derivative $\operatorname{Tr} \mathcal{L}(s)=-\frac{d}{d s} \log F(s)$ of the Fredholm determinant:

$$
F(\beta, s)=\exp \left(-\sum_{p, r} \frac{e^{\left(h_{p}+s T_{p}\right) r}}{r\left|\operatorname{det}\left(1-\mathbf{J}_{p}^{r}\right)\right|} \Delta_{p, r}(\beta)\right) .
$$

This determinant is the central result of this paper. Its zeros correspond to the eigenvalues of the evolution operator (6), and can be evaluated by standard cycle expansion methods [3,17].

In the "thermodynamic" formalism 12,13, 14, for classical chaotic systems, and in the Gutzwiller semi-classical description of systems with chaotic classical counterpart [5], $\beta$ is a parameter which plays the role of "inverse temperature", and $h_{p}$ is the integral of some weight function $h(x)$ evaluated along the prime periodic orbit $p$. In the semi-classical quantization case [5]

$$
\beta=1 / 2, \quad h_{p}=i S_{p} / \hbar+i \nu_{p} \pi / 2, \quad s=0,
$$

where $S_{p}$ is the action of the periodic orbit, and $\nu_{p}$ its Maslov index. The classical correlation spectra are given by $\beta=1$ and $h_{p}=0$. 
The simplest application of (10) is to 3-dimensional hyperbolic Hamiltonian flows (higher dimensions need further evolution operators, for outer products of vectors rather than single vectors). In this case $\Lambda_{1}=1 / \Lambda_{2}=\Lambda$, and the Fredholm determinant is given by

$$
\begin{aligned}
F_{\sigma}(\beta, s) & =\exp \left(-\sum_{p, r} \frac{\sigma_{p}^{r}}{r\left|\Lambda_{p}^{r}\right|} \frac{e^{r\left(h_{p}+s T_{p}\right)}}{\left(1-1 / \Lambda_{p}^{r}\right)^{2}} \Delta_{p, r}(\beta)\right) \\
\Delta_{p, r}(\beta) & =\frac{\left|\Lambda_{p}^{r}\right|^{-\beta+1}}{1-1 / \Lambda_{p}^{2 r}}+\frac{\left|\Lambda_{p}^{r}\right|^{\beta-3}}{1-1 / \Lambda_{p}^{2 r}} .
\end{aligned}
$$

The extra multiplicative factor is set to the eigenvalue $\operatorname{sign} \sigma=\Lambda /|\Lambda|$ for $F_{-}$, and to $\sigma=1$ for $F_{+}$; this will be used below.

The Gutzwiller-Voros zeta function corresponds to setting $\Delta_{p, r}=\left|\Lambda_{p}^{r}\right|^{1 / 2}\left(1-1 / \Lambda_{p}^{r}\right)$, and the "quantum Fredholm determinant" [2] is obtained by setting $\Delta_{p, r}=$ $\left|\Lambda_{p}^{r}\right|^{1 / 2}$ in (12). The practical advantage of (10) over the more familiar Gutzwiller-Voros and Ruelle type zeta functions was demonstrated by detailed numerical studies [3] of the related quantum Fredholm determinant [2]. In the systems studied, the quantum Fredholm determinant appeared to be entire for all practical purposes; only the most recent numerical investigations [18] reveal poles absent in (10), see fig. 1 .

It can be shown 18] that the Fredholm determinant obtained by keeping only one of the terms in the sum in (9) is entire. This enables us to show that the GutzwillerVoros zeta function $Z(E)$ for Axiom A flows is meromorphic in the complex $E$ plane, as it can be written as a ratio of entire functions; for 2-dimensional Hamiltonian systems

$$
Z(E)=\frac{F_{+}\left(\frac{1}{2}, E\right) F_{-}\left(\frac{7}{2}, E\right)}{F_{-}\left(\frac{3}{2}, E\right) F_{+}\left(\frac{5}{2}, E\right)},
$$

where $F_{\sigma}$ includes only the first term in the $\Delta_{p, r}$ sum (12). The zeros of the Gutzwiller-Voros zeta function coincide with the ones obtained from $F_{+}\left(\frac{1}{2}, E\right)$. Such relations follow by inserting into $\Delta_{p, r}$ identities like

$$
1=\frac{1}{1-1 / \Lambda^{r}}-\frac{1}{\Lambda^{r}} \frac{1}{\left(1-1 / \Lambda^{r}\right)} .
$$

( $\sigma$ weight in $(12)$ is needed to account for the $1 / \Lambda=\sigma /|\Lambda|$ term in the above indentity).

We illustrate a choice of the $g(\xi)$ function and construction of the $R$ dynamics (5) by a simple explicit example: 2-dimensional Hamiltonian dynamics reduced to a 2-dimensional Poincaré section return map $x_{i+1}=f\left(x_{i}\right)$. The stability matrix of cycle $p$ is a product of the $[2 \times 2]$ stability matrices

$$
\mathbf{J}_{j}=\left(\begin{array}{cc}
A_{j} & B_{j} \\
C_{j} & D_{j}
\end{array}\right)
$$

where $A_{j}=\partial f_{1}\left(x_{i}\right) / \partial x_{1}$, and so on. Assume the signed norm (3) and multiply an initial unit vector by the first stability matrix in the product. The resulting vector can be written as

$$
\mathbf{J}_{1}\left(\begin{array}{c}
\kappa_{1} \\
1
\end{array}\right)=\left(C_{1} \kappa_{1}+D_{1}\right)\left(\begin{array}{c}
\frac{A_{1} \kappa_{1}+B_{1}}{C_{1} \kappa_{1}+D_{1}} \\
1
\end{array}\right)
$$

Hence the dynamics acts on the unit vectors as a rational fraction transformation

$$
\kappa_{k+1}=R\left(x_{k}, \kappa_{k}\right)=\frac{A_{k} \kappa_{k}+B_{k}}{C_{k} \kappa_{k}+D_{k}},
$$

with the signed norm (4) of the iterated vector given by

$$
\Lambda^{n_{p}}\left(x_{1}, \kappa_{1}\right)=\prod_{i=1}^{n_{p}}\left(C_{i} \kappa_{i}+D_{i}\right) .
$$

(In the case of 2-dimensional billiards, $\kappa$ is the Bunimovich-Sinai curvature [19]). For a periodic orbit $\kappa_{n_{p}}=\kappa_{0}$, the unit vector is an eigenvector of the stability matrix, and the corresponding eigenvalue is $\Lambda_{p}=\prod\left(C_{i} \kappa_{i}+D_{i}\right)$. For 3-dimensional hyperbolic flows there are two distinct $\kappa_{p, i}$ values, one for the expanding, and the other for the contracting eigenvalue. The derivative of $R^{n_{p}}$ is easily evaluated;

$$
\frac{\partial}{\partial \kappa} R^{n_{p}}\left(\kappa_{1}, x_{1}\right)=\prod_{i=1}^{n_{p}} R^{\prime}\left(\kappa_{i}, x_{i}\right)=\prod_{i=1}^{n_{p}} \frac{1}{\left(C_{i} \kappa_{i}+D_{i}\right)^{2}}
$$

(we have used $\operatorname{det}\left(\mathbf{J}_{i}\right)=1$ ) and we again obtain the trace formula (12).

In conclusion, we have constructed a classical evolution operator for semi-classical quantization, and derived a new determinant for periodic orbit quantization of chaotic dynamical systems. The main virtue of the determinant $(10)$ is that the theorem of H.H. Rugh [20], applicable to multiplicative evolution operators such as (6), implies that this determinant should be entire for Axiom A flows, $i e$. free of poles in the entire complex $s$ or complex energy plane. One consequence of this general result is that the cycle expansions of the new Fredholm determinant should converge faster than exponentially. Our numerical tests support above claims [18]; one example is given in fig. 11 which demonstrates that the new determinant is analytic to the limit of numerical precision of current cycle expansions, and well beyond both the Gutzwiller-Voros zeta function and the quantum Fredholm determinant.

\section{ACKNOWLEDGMENTS}

G.V. is indebted to D. Szász and A. Krámli for pointing out the importance of the Bunimovich-Sinai curvatures, to the Széchenyi Foundation and OTKA F4286 for the support, and to the Center for Chaos and Turbulence 
Studies, Niels Bohr Institute, for hospitality. P.C. thanks the Carlsberg Fundation for support.

* On leave from Eötvös University, Budapest, Hungary.

[1] B. Eckhardt and G. Russberg, Phys. Rev. E 47, 1578 (1993).

[2] P. Cvitanović and P.E. Rosenqvist, Proc. SISSA Trieste workshop "From Classical to Quantum Chaos", July 1992; N. Cimento, to appear.

[3] P. Cvitanović, P.E. Rosenqvist, H.H. Rugh, and G. Vattay, submitted to CHAOS (1993).

[4] A. Voros, J. Phys. A 21, 685 (1988).

[5] M.C. Gutzwiller, Chaos in Classical and Quantum Mechanics (Springer, New York 1990).

[6] P. Gaspard and S.A. Rice, J. Chem. Phys. 90, 2225 (1989); 90, 2242 (1989); 90, 2255 (1989).

[7] P. Cvitanović and B. Eckhardt, Phys. Rev. Lett. 63, 823 (1989).

[8] P. Gaspard and D. Alonso Ramirez, Phys. Rev. A 45, 8383 (1992).

[9] G. Bennettin, L. Galgani and J.-M. Strelcyn, Meccanica 15, 9 (1980).

[10] A similar extension to the tangent space has been introduced by E. Gozzi, M. Reuter and W.D. Thacker, Phys. Rev. D 40, 3363 (1989) and other authors, but with rather different motives and applications in mind.

[11] V.I. Arnold, Ordinary Differential Equations (MIT Press, Cambridge, Mass. 1978)

[12] D. Ruelle, Statistical Mechanics, Thermodynamic Formalism (Addison-Wesley, Reading MA, 1978).

[13] T.C. Halsey, M.H. Jensen, L.P. Kadanoff, I. Procaccia and B.I. Shraiman, Phys. Rev. A107, 1141 (1986).

[14] P. Szépfalusy, T. Tél, A. Csordás and Z. Kovács, Phys. Rev. A 36, 3525 (1987).

[15] P. Cvitanović, ed., Periodic Orbit Theory - theme issue, CHAOS 2, 1-158 (1992).

[16] P. Cvitanović and B. Eckhardt, J. Phys. A 24, L237 (1991).

[17] R. Artuso, E. Aurell and P. Cvitanović, Nonlinearity 3, 325 (1990).

[18] G. Vattay, in preparation.

[19] L. Bunimovich and Ya.G. Sinai, Comm. Math. Phys. 78, 247 (1980); 78, 479 (1980); Erratum, ibid. 107, 357 (1986).

[20] H.H. Rugh, Nonlinearity 5, 1237 (1992). See also ref. [3] for a discussion of the applicability of the theorem.
FIG. 1. The leading semi-classical resonances in the $k$ complex wave-number plane $(\times)$ for the determinant (10), compared with $(\square)$ the zeros of the "quantum Fredholm determinant" 2]3. While the quantum Fredholm determinant has a finite region of analyticity (the bottom line of zeros reflects a pole expected at $\operatorname{Im}(k)=-4.559843 \ldots$, indicated by the dashed line labeled "QF"), the new determinant shows no numerical indication of any poles, and enables us to reach resonances deeper down in the complex plane. The Gutzwiller-Voros determinant is reliable only down to the line labeled "GV", $\operatorname{Im}(k)=-2.491905 \ldots$, the upper bound on the poles of determinant $F_{-}\left(\frac{3}{2}, E\right)$ in $(13)$. The dynamical system tested is the Hamiltonian Hénon map $x_{k+1}=1-a x_{k}^{2}-x_{k-1}$ at $a=20$, with cycle expansions truncated to cycles up to period 18. We take as action $S_{p} / \hbar=k n_{p}$, and as the Maslov phase $\nu_{p}=2 n_{p}$ (this is a normal-form model of a 3 -disc repeller, see ref. [3]). 